\title{
Justification of Unruptured Intracranial Aneurysm Repair: A Single-Center Experience
}

\author{
T. Ishibashi, Y. Murayama, T. Saguchi, M. Ebara, H. Arakawa, K. Irie, H. Takao, and T. Abe
}

\begin{abstract}
BACKGROUND AND PURPOSE: Whether to treat UIAs is controversial. The aim of the study was to compare the clinical outcome of patients with UIAs who were either treated conservatively or preventively.
\end{abstract}

MATERIALS AND METHODS: Patients with UIAs referred to our institution were prospectively enrolled in the study. Data collected included baseline characteristics, aneurysmal features, and procedural and follow-up information. Preventive treatment was recommended if the aneurysm was larger than $5 \mathrm{~mm}$ and was considered safely treatable. Endovascular surgery was the first-line therapy if the aneurysmal shape was appropriate for coiling.

RESULTS: From January 2003 through April 2008, a total of 879 patients with 1110 UIAs were enrolled; 325 patients with 369 UIAs (mean size, $7.8 \mathrm{~mm}$ ) were treated (treatment group), and 603 patients with 741 UIAs (mean size, $4.4 \mathrm{~mm}$ ) were managed conservatively (observation group). Mean follow-up was 692.5 days (1405.5 person-years). In the observation group, 26 aneurysms (3.5\%) had ruptured (1.8\% per year; 1405.5 person-years), 10 patients died, and 7 were disabled (mRS, 3-6: 2.8\%). Aneurysmal size was a significant risk factor for rupture ( $P=.001)$. The treatment group included aneurysms treated either with coiling $(n=315)$, clipping $(n=32)$, or a combined approach $(n=9) ; 1$ patient died, and 3 were disabled (mRS, 3-6: 1.2\%). Therapeutic intervention was equal (UIAs of all sizes) or superior (UIAs $>5 \mathrm{~mm} ; P=.025$ ) to conservative management.

CONCLUSIONS: Treatment of UIAs was justified in aneurysms larger than $5 \mathrm{~mm}$, and EVS can be safely applied to nearly $90 \%$ of UIAs.

ABBREVIATIONS: EVS = endovascular surgery; ISUIA, International Study of Unruptured Intracranial Aneurysms; MC = microsurgical clipping; mRS = modified Rankin Scale; UIA = unruptured intracranial saccular aneurysm

W hether to treat UIAs depends on various factors, including the risks associated with the natural history of UIAs and those associated with preventive treatment. To date, there are no data from prospective, randomized trials comparing conservative management of UIAs with preventive treatment by use of $\mathrm{MC}$ or EVS. The ISUIA ${ }^{1,2}$ found a low overall rupture rate and appreciable treatment risks, which could be interpreted to mean that preventive treatment is rarely justified. Recent large studies also reported relatively low rupture rates for small UIAs., ${ }^{3,4}$ Since the publication of these studies, the management strategy for UIAs has tended to be conservative. However, larger UIAs, which are

Received September 7, 2012; accepted after revision November 10.

From the Division of Endovascular Neurosurgery, Department of Neurosurgery (T.I., Y.M., M.E., H.A., K.I., H.T.), and Department of Neurosurgery (T.A.), The Jikei University School of Medicine, Tokyo, Japan; and Department of Neurosurgery (T.S.), Japanese Red Cross Medical Center, Tokyo, Japan.

Please address correspondence to Toshihiro Ishibashi, MD, Division of Endovascular Neurosurgery, Department of Neurosurgery, The Jikei University School of Medicine, 3-25-8 Nishi-shinbashi, Minato-Ku, Tokyo 105-8461, Japan; e-mail: t-ishibashi@jikei.ac.jp

- Indicates open access to non-subscribers at www.ajnr.org

http://dx.doi.org/10.3174/ajnr.A3470 associated with a higher risk for rupture, usually benefit from preventive treatment. It is still unclear on how to treat the aneurysm based on this size. The aim of this study was to compare the clinical outcome of patients with UIAs who were either treated conservatively or treated preventively.

\section{MATERIALS AND METHODS}

\section{Selection and Description of Patients}

Data and demographics on all patients with UIAs referred to our institution were prospectively collected in our data base. Patients were not enrolled if they had 1) fusiform, traumatic, or mycotic aneurysms; or 2) a UIA treated before entry into the study. Data entered into the data base included baseline characteristics (including demographics, a history of SAH, and mRS), aneurysmal features (including size, location, and morphologic patterns), and procedural and follow-up information (including aneurysmal rupture).

\section{Imaging and Management Strategies}

We measured UIAs by using 3D-CTA (Sensation 16; Siemens, Erlangen, Germany), except for patients who had contraindications related to contrast material. The $3 \mathrm{D}$ acquisition data were sent to 


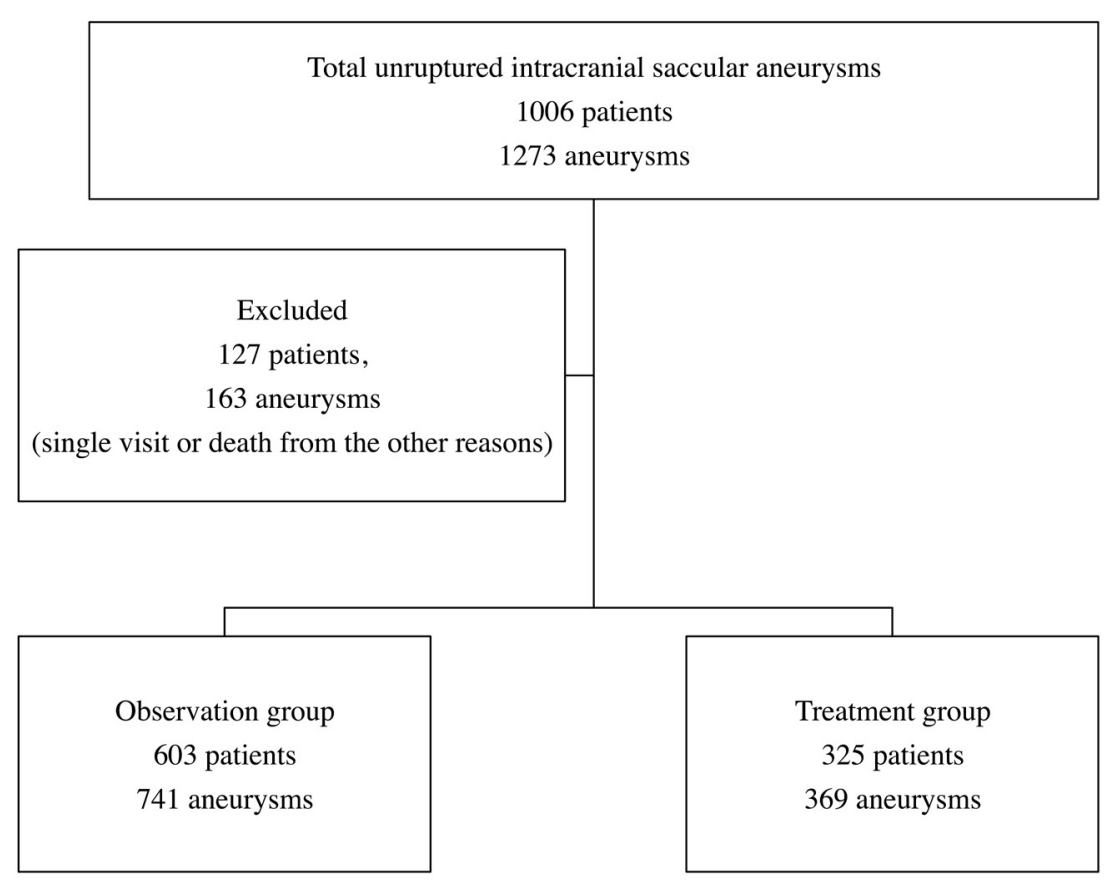

FIG 1. Flow chart of patient assignments and treatments.

Table 1: Characteristics of study population and UIAs

\begin{tabular}{|c|c|c|c|c|}
\hline & \multirow[b]{2}{*}{ No. of Patients } & \multicolumn{2}{|c|}{ UIAs ( $n=\%)$} & \multirow[b]{2}{*}{$P$ Value } \\
\hline & & Observed & Treated & \\
\hline & 879 & $(741 ; 66.8 \%)$ & $(369 ; 33.3 \%)$ & \\
\hline Mean size of UIAs (mm) & & $4.4 \pm 0.1$ & $7.8 \pm 0.2$ & \\
\hline Female sex (\%) & $617(70.2)$ & $527(71.1)$ & $284(77)$ & .039 \\
\hline \multicolumn{5}{|l|}{ No. of UIAs } \\
\hline Single & & $422(57.0)$ & $227(61.5)$ & .146 \\
\hline Multiple & & $319(43.0)$ & $142(38.5)$ & \\
\hline $\begin{array}{l}\text { UIA associated with history of SAH from } \\
\text { a separate ruptured aneurysm }\end{array}$ & & 30 & 36 & $<.001$ \\
\hline \multicolumn{5}{|l|}{ Size of UIAs } \\
\hline Small $(<5 \mathrm{~mm})$ & & $534(72.1)$ & $65(17.6)$ & $<.001$ \\
\hline Medium (5-9.9 mm) & & $175(23.6)$ & $228(61.8)$ & $<.001$ \\
\hline Large (10-24.9 mm) & & $30(4)$ & $72(19.5)$ & $<.001$ \\
\hline Giant (> $25 \mathrm{~mm}$ & & $2(0.3)$ & $4(1)$ & .081 \\
\hline \multicolumn{5}{|l|}{ Location of UIAs } \\
\hline Internal carotid artery & & $319(43)$ & $205(55.6)$ & $<.001$ \\
\hline Anterior cerebral artery & & $136(18.4)$ & 59 (16) & .329 \\
\hline Middle cerebral artery & & $213(28.7)$ & $66(17.9)$ & $<.001$ \\
\hline Vertebrobasilar artery & & $73(9.9)$ & 39 (10.6) & .708 \\
\hline
\end{tabular}

a 3D workstation (Aquarius, TeraRecon, San Mateo, California) for precise aneurysmal measurements, which were conducted independently by an experienced radiology technologist. Aneurysmal size was classified according to the national guidelines for the management of UIAs of the Japanese Society for Detection of Asymptomatic Brain Diseases ${ }^{5}$ : small, up to $4.9 \mathrm{~mm}$ in diameter; medium, 5-9.9 mm in diameter; large, larger than $10 \mathrm{~mm}$; and giant, larger than $25 \mathrm{~mm}$.

Following the national guidelines for the management of UIAs, ${ }^{5}$ preventive treatment with EVS or MC was recommended if 1) the aneurysm was larger than $5 \mathrm{~mm}$ and was considered safely treatable either with EVS or MC, 2) the patient had multiple aneurysms and one had previously ruptured, or 3) the patient had a family history of SAH. EVS was the first-line therapy if the aneurysmal shape and vascular anatomy were appropriate for coil embolization. All MC and EVS procedures were performed by a single neurovascular team. Preprocedural and immediate postprocedural 3D angiography were performed by use of a high-end 3D digital subtraction angiography unit. ${ }^{6}$

\section{Follow-Up}

Patients treated conservatively were scheduled for consultation and 3D-CTA follow-up every 6 months. Because 3DCTA was more sensitive than MRA based on our previous study, ${ }^{7}$ we selected 3D-CTA for follow-up. Patients treated with EVS were scheduled for consultation and MRA follow-up at 3, 6 , and 12 months after treatment; all patients treated with MC or EVS were scheduled for follow-up angiography at 12 months after treatment. If the aneurysm was stable, then annual MRA and MR imaging were scheduled for all patients. In addition, 3D-CTA was scheduled for the patients treated with MC. Neurologic status was measured with mRS at each follow-up assessment.

\section{Adverse Events}

Neurologic status at 30 days after an adverse event was assessed by mRS. Aneurysmal rupture alone without subsequent neurologic deterioration (ie, only with severe headache after SAH) was not considered a morbidity; only adverse events with subsequent neurologic deterioration (mRS, 1-6) 30 days after the adverse event were evaluated.

\section{Statistical Analysis}

Data analysis of adverse events (incidence of rupture or complication) was calculated per aneurysm. All statistical analyses were performed using STATA 9.2 (STATA Corp., College Station, Texas). We compared categoric variables by using the Fisher exact 2-tailed test, the Pearson $\chi^{2}$ test, or the test for determining linear trend. Continuous variables were compared among groups by use of the Mann-Whitney $U$ test or the Student $t$ test. For life-table analysis and the Cox proportional hazards regression model, each patient was observed to the time of $\mathrm{SAH}$, death from causes other than $\mathrm{SAH}$, or to the last possible follow-up contact. We calculated the average annual incidence of $\mathrm{SAH}$ by determining the number of first-event SAHs divided by the number of person-years of follow-up. Cumulative rates of probability of morbidity and mortality rates were estimated by 
Table 2: Observation group: risk for rupture associated with size, history of SAH, multiplicity, sex, and location of UIA

\begin{tabular}{|c|c|c|c|c|c|}
\hline Variable & $\begin{array}{c}\text { No. Ruptured } \\
\text { Aneurysms }\end{array}$ & $\begin{array}{l}\text { No. Total } \\
\text { UIAs }\end{array}$ & $\begin{array}{c}\text { Annual Rupture } \\
\text { Rate (\%) }\end{array}$ & $\begin{array}{c}\text { Hazard Ratio } \\
(95 \% \mathrm{Cl})\end{array}$ & $\begin{array}{c}P \\
\text { Value }\end{array}$ \\
\hline \multicolumn{6}{|l|}{ Size of aneurysm (mm) } \\
\hline Total & 26 & 741 & 1.8 & & \\
\hline Small (<5 mm) & 7 & 534 & 0.7 & 1 (reference) & \\
\hline Medium (5-9.9 mm) & 10 & 175 & 2.7 & $3.4(1.3-9.0)$ & .014 \\
\hline Large (10-24.9 mm) & 7 & 30 & 8.1 & $10.8(3.7-32.0)$ & $<.001$ \\
\hline Giant (> $25 \mathrm{~mm})$ & 2 & 2 & 52.9 & $62.6(12.7-308.9)$ & $<.001$ \\
\hline \multicolumn{6}{|l|}{ Location of aneurysm } \\
\hline Internal carotid artery & 9 & 319 & 1.6 & 1 (reference) & \\
\hline Anterior cerebral artery & 3 & 136 & 1.2 & $0.8(0.2-3.2)$ & .759 \\
\hline Middle cerebral artery & 7 & 213 & 1.7 & $1.1(0.5-3.7)$ & .827 \\
\hline Vertebrobasilar artery & 7 & 73 & 3.8 & $2.3(1.0-6.2)$ & .104 \\
\hline \multicolumn{6}{|l|}{ Circulation location } \\
\hline Anterior circulation & 19 & 668 & 1.6 & 1 (reference) & \\
\hline Posterior circulation & 7 & 73 & 3.8 & $2.3(0.9-5.7)$ & .068 \\
\hline \multicolumn{6}{|l|}{ History of SAH } \\
\hline No & 19 & 711 & 2.0 & 1 (reference) & \\
\hline Yes & 7 & 30 & 9.6 & $6.1(2.5-15.0)$ & $<.001$ \\
\hline Small $(<5 \mathrm{~mm})$ & 3 & 23 & 5.2 & & \\
\hline Medium (5-9.9 mm) & 2 & 5 & 14.1 & & \\
\hline Large (10-24.9 mm) & 2 & 2 & 166.7 & & \\
\hline \multicolumn{6}{|l|}{ Multiplicity of aneurysm } \\
\hline Single & 12 & 432 & 1.5 & 1 (reference) & \\
\hline Multiple & 14 & 309 & 2.2 & $1.6(0.7-3.5)$ & .254 \\
\hline
\end{tabular}

Note:- $\mathrm{Cl}$ indicates confidence interval.

Table 3: Clinical outcome of enrolled patients

\begin{tabular}{ccccc}
\hline \multicolumn{5}{c}{ No. of Patients (No. of UIAs) } \\
\cline { 2 - 5 } mRS & $\begin{array}{c}\text { Observation Group } \\
(\boldsymbol{n}=603)\end{array}$ & $\%$ & $\begin{array}{c}\text { Treatment Group } \\
(\boldsymbol{n}=\mathbf{3 2 5})\end{array}$ & $\%$ \\
\hline 0 & $585(723)$ & 97 & $314(358)$ & 96.6 \\
1 & $1(1)$ & 0.17 & $4(4)$ & 1.2 \\
2 & $0(0)$ & 0 & $3(3)$ & 0.9 \\
3 & $3(3)$ & 0.5 & $2(2)$ & 0.6 \\
4 & $2(2)$ & 0.33 & $1(1)$ & 0.3 \\
5 & $2(2)$ & 0.33 & $0(0)$ & 0 \\
6 & $10(10)$ & 1.67 & $1(1)$ & 0.3 \\
\hline
\end{tabular}

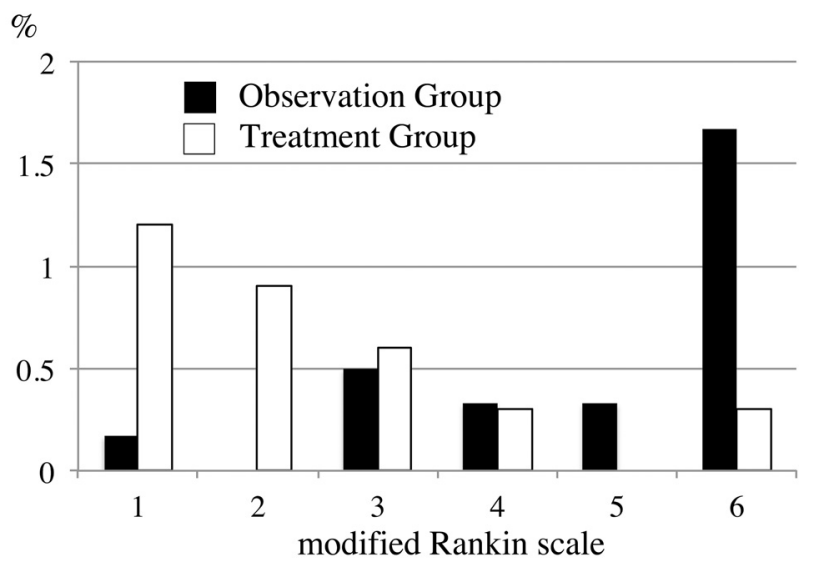

FIG 2. Proportion of mRS scores in the observation group and the treatment group. Clinical outcome tends to be worse in the observation group compared with the treatment group.

use of the Kaplan-Meier product-limit method. Statistical differences were determined by the Cox proportional hazard model by treatment. were treated.

\section{RESULTS}

From January 2003 through April 2008, a total of 1006 patients with 1273 UIAs were referred to our institution and their data prospectively entered into our data base. Of these, 127 patients (12.6\%) with 163 UIAs (12.8\%) were excluded because they had only a single visit, were lost to followup, or died from other causes. Therefore, the study population consisted of $879 \mathrm{pa}$ tients and 1110 UIAs (Fig 1). Patient and aneurysmal characteristics of the study population are summarized in Table 1 . The ratio of women to men was almost 2:1. Ages ranged from 17 to 86 years, with an average of 59.2 years. Three hundred and twenty-five patients with 369 UIAs $(33.2 \%)$ were treated either with EVS, MC, or both (treatment group): 287 patients with 315 UIAs (85.4\%) with EVS only, 29 patients with 32 UIAs $(8.7 \%)$ with MC only, and 9 patients with multiple aneurysms received both EVS and MC. In the treatment group, the mean size of the aneurysm was $7.8 \mathrm{~mm}$, and the smallest aneurysmal size was 2.0 $\mathrm{mm}$. Six hundred and three patients with 741 UIAs (66.8\%) received conservative management (observation group). The mean follow-up was 692.5 days (1405.5 person-years). The mean aneurysmal size in the observation group was $4.4 \mathrm{~mm}$. Of all 1110 UIAs, 599 (54\%) were small, 403 (36.3\%) medium, $102(9 \%)$ large, and $6(0.5 \%)$ giant. The proportion of UIAs treated was increased per the size of the aneurysm: small, $11 \%$; medium, 56.6\%; large, 70.1\%; and giant, 66.6\%. Sixty-six UIAs were associated with a history of SAH from a separate aneurysm (group 2 in the ISUIA); of these, 30 were observed and 36

\section{Adverse Events and Clinical Outcome}

In the observation group, 26 aneurysms (3.5\%) were ruptured ( $1.8 \%$ per year; 1405.5 person-years) during follow-up; the details are summarized in Table 2 . The annual rupture rate per aneurysmal size was small, 0.7\%; medium, 2.7\%; large, $8.1 \%$; and giant, $52.9 \%$. The incidence of rupture correlated strongly with aneurysmal size. The annual rupture rate for UIAs associated with a history of SAH from a separate aneurysm (ISUIA group 2) was higher than that for UIAs not associated with a history of SAH: $2.0 \%$ vs $9.6 \%$, respectively. In UIAs associated with a history of $\mathrm{SAH}$, the annual rupture rate per aneurysmal size was small, $5.2 \%$; medium, 14.1\%; and large, $166.7 \%$.

The time to aneurysmal rupture from the first day of observation was between 10 and 1461 days. Eighteen patients in the observation group deteriorated neurologically (mRS, 1-6) after aneurysmal rupture (Table 3 ). Ten patients died, and 7 patients were disabled (mRS, 3-6;2.8\%). The reasons for choosing conservative management for the 26 patients with UIAs that subsequently ruptured included advanced age ( 5 patients), an- 
Table 4: Treatment group: details of adverse events in patients with neurologic deterioration

\begin{tabular}{|c|c|c|c|c|c|}
\hline Procedure & $\begin{array}{l}\text { UIA Size } \\
\text { (mm) }\end{array}$ & $\begin{array}{c}\text { Classification of } \\
\text { Event }\end{array}$ & Details of Event & Symptoms & $\begin{array}{l}\mathrm{mRS} \text { at } \\
30 \text { Days }\end{array}$ \\
\hline EVS & Medium (7.4) & Thromboembolic & Infarct & $\begin{array}{l}\text { Fine movement } \\
\text { loss }\end{array}$ & 1 \\
\hline EVS & Medium (7.2) & Thromboembolic & Infarct & & 1 \\
\hline EVS & Large (20.9) & Thromboembolic & $\begin{array}{l}\text { Coil mass } \\
\text { compression }\end{array}$ & Right hemianopia & 1 \\
\hline EVS & Large (23) & Thromboembolic & Infarct & Visual field defect & 1 \\
\hline$M C$ & Medium (7.4) & Thromboembolic & Infarct & Memory loss & 2 \\
\hline EVS & Large (10.9) & Thromboembolic & Infarct & & 2 \\
\hline EVS & Medium (6.7) & Hemorrhagic & $\begin{array}{c}\text { Acute subdural } \\
\text { hematoma }\end{array}$ & & 2 \\
\hline EVS & Large (14) & Thromboembolic & Infarct & Aphasia & 3 \\
\hline EVS & Medium (6) & Hemorrhagic & $\begin{array}{l}\text { SAH after } \\
\text { embolization }\end{array}$ & $\mathrm{SAH}$ & 3 \\
\hline EVS & Medium (5.6) & Hemorrhagic & $\begin{array}{l}\text { Periprocedural } \\
\text { rupture }\end{array}$ & $\begin{array}{c}\text { SAH, intracerebral } \\
\text { hemorrhage }\end{array}$ & 4 \\
\hline EVS & Large (14.3) & Hemorrhagic & $\begin{array}{l}\text { SAH after } \\
\text { embolization }\end{array}$ & $\mathrm{SAH}$ & 6 \\
\hline
\end{tabular}

Table 5: Treatment group: outcome in 325 patients

\begin{tabular}{|c|c|c|c|c|}
\hline & $\begin{array}{c}\text { EVS Alone } \\
(n=287)\end{array}$ & $\begin{array}{l}\text { MC Alone } \\
(n=29) \\
\end{array}$ & $\begin{array}{c}\text { EVS and MC } \\
\text { Combined }(n=9)\end{array}$ & $\begin{array}{c}\text { Total } \\
(n=325)\end{array}$ \\
\hline & $n(\%)$ & $n(\%)$ & $n(\%)$ & $n(\%)$ \\
\hline \multicolumn{5}{|l|}{ mRS $0-2$ at 30 days } \\
\hline Thromboembolic complications & $19(6.6)$ & 4 (13.7) & 0 & $23(7.1)$ \\
\hline Hemorrhagic complications & $5(1.7)$ & $4(13.7)$ & 0 & $9(2.8)$ \\
\hline Puncture site & $3(1.0)$ & 0 & 0 & $3(0.9)$ \\
\hline Other & $1(0.3)$ & $1(3.4)$ & 0 & $2(0.6)$ \\
\hline \multicolumn{5}{|l|}{ mRS $3-6$ at 30 days } \\
\hline Thromboembolic complications & $1(0.3)$ & 0 & 0 & $1(0.3)$ \\
\hline Hemorrhagic complications & $3(1.0)$ & 0 & 0 & $3(0.9)$ \\
\hline Morbidity (mRS 3-5) at 30 days & & & & $3(0.9)$ \\
\hline Small $(<5 \mathrm{~mm})$ & 0 & 0 & 0 & 0 \\
\hline Medium (5-9.9 mm) & $2(0.7)$ & 0 & 0 & $2(0.6)$ \\
\hline Large (10-24.9 mm) & $1(0.4)$ & 0 & 0 & $1(0.3)$ \\
\hline Giant $(>25 \mathrm{~mm})$ & 0 & 0 & 0 & 0 \\
\hline Mortality at 30 days & & & & $1(0.3)$ \\
\hline Small (<5 mm) & & 0 & 0 & 0 \\
\hline Medium (5-9.9 mm) & 0 & 0 & 0 & 0 \\
\hline Large (10-24.9 mm) & $1(0.4)$ & 0 & 0 & $1(0.3)$ \\
\hline Giant $(>25 \mathrm{~mm})$ & 0 & 0 & 0 & 0 \\
\hline Morbidity and mortality at 30 days & & & & $4(1.2)$ \\
\hline Patients with small aneurysms & & & & 0 \\
\hline Patients with nonsmall aneurysms & & & & $4(1.5)$ \\
\hline
\end{tabular}

cations occurred in 8 EVSs and 4 MCs. Fourteen of the 20 EVS thromboembolic complications were transient ischemic events and did not affect the mRS score at 30 days; in the other 6 patients, 4 had an mRS score of 1,1 had a score of 2 , and 1 had a score of 3 . Of the $4 \mathrm{MC}$ thromboembolic complications, 3 did not affect the mRS score, but 1 patient had an ischemic stroke resulting from a perforator injury or venous infarction and had an mRS score of 2. Overall, thromboembolic complications resulting in an mRS score of $3-6$ were found in 1 patient $(0.3 \%)$. Hemorrhagic complications occurred in 8 EVSs and $4 \mathrm{MCs}$ in the treatment group. Of the 12 hemorrhagic complications, 8 did not affect the mRS score at 30 days: 2 aneurysmal perforations during the procedure, 2 vessel perforations, and 4 asymptomatic intracranial hemorrhages. Four patients with hemorrhagic complications continued to have neurologic deficits at 30 days ( 1 each; mRS, 2, 3, 4, and 6$)$. The details of the 11 patients $(3.4 \%)$ in the treatment group who deteriorated neurologically (mRS, 1-6) at 30 days are summarized in Table 4 . Of these 11 patients, 1 died and 3 remained moderately to severely disabled. Overall, morbidity and mortality rates at 30 days were $0.9 \%$ and $0.3 \%$, respectively, all in patients with nonsmall ( $\geq 5 \mathrm{~mm}$ ) UIAs (Table 5). Morbidity and mortality rates at 30 days in patients with small UIAs and nonsmall UIAs were $0 \%$ and $1.5 \%$, respectively. Although no clinical adverse events occurred in the treatment group for small aneurysms $(<5$ $\mathrm{mm}$ ), overall clinical outcomes for the treatment and observation groups were identical for all UIA sizes (Fig 3A). Treatment benefit was confirmed in atomic difficulty (5 patients), refused treatment ( 5 patients), aneurysms very small in size (4 patients), rupture before treatment ( 4 patients), and rupture during the period of imaging work-up (3 patients).

Clinical outcomes of enrolled patients are shown in Fig 2 and Table 3. Overall, the percentage of patients with an mRS score of 0 at 30 days was $97 \%$ in the observation group and $96.6 \%$ in the treatment group (Table 3). However, when an adverse event occurred, the clinical outcome tended to be worse in the observation group compared with the treatment group. Mortality rate was higher in the observation group than in the treatment group.

Details of clinical outcomes for the treatment group are shown in Table 4. In the treatment group, thromboembolic complications occurred in 20 EVSs and $4 \mathrm{MCs}$, and hemorrhagic compli-
UIAs larger than $5 \mathrm{~mm}$ if an mRS of 3 was considered an adverse event $(P=.025)$ (Fig $3 B)$.

The probability of morbidity and mortality did not yield a significant difference between the treatment group and the observation group in UIAs less than $5 \mathrm{~mm}$ (Fig 3C).

\section{DISCUSSION}

There are no data from randomized studies comparing treatment outcomes with the natural history of UIAs. The Trial on Endovascular Aneurysm Management ${ }^{8}$ was the first randomized controlled trial to compare conservative management of UIAs with endovascular treatment. The primary endpoint was mortality and morbidity (defined as an $\mathrm{mRS} \geq 3$ ) from intracranial hemorrhage or treatment. Secondary endpoints included the incidence of hemorrhagic events, morbidity related to endovascular coiling, 


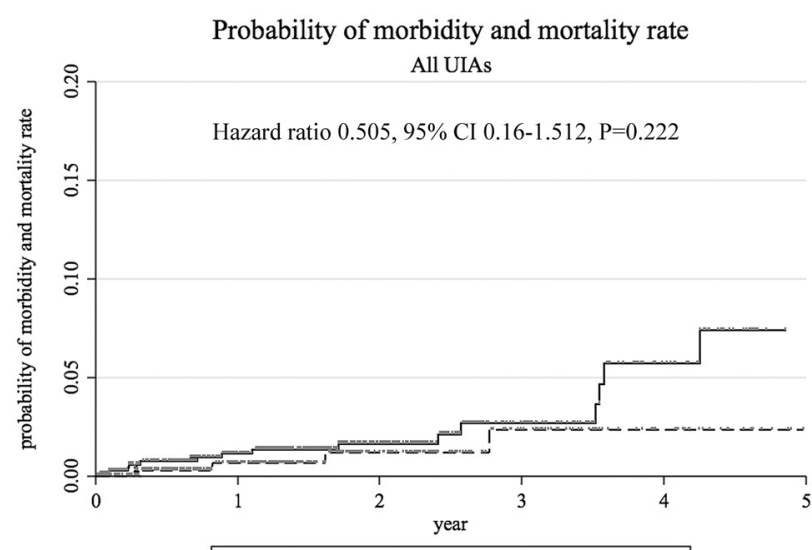

A

- Observation group ----- Treatment group

Probability of morbidity and mortality rate

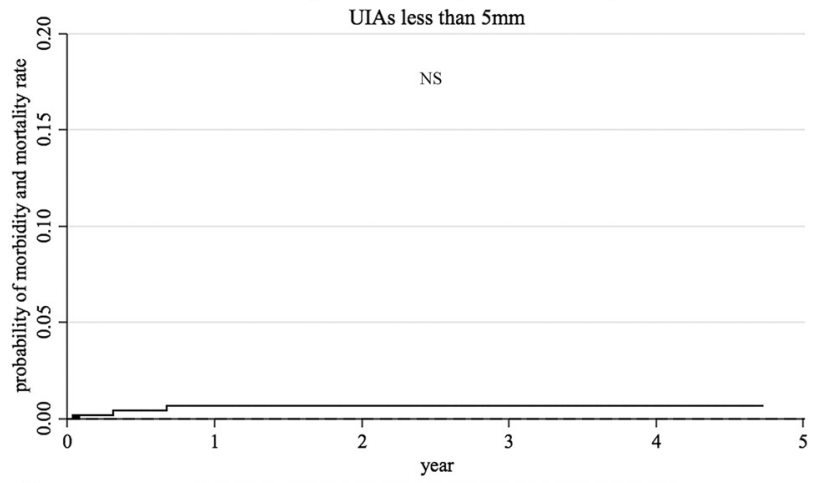

C

Observationgroup - - - - - Treatment group

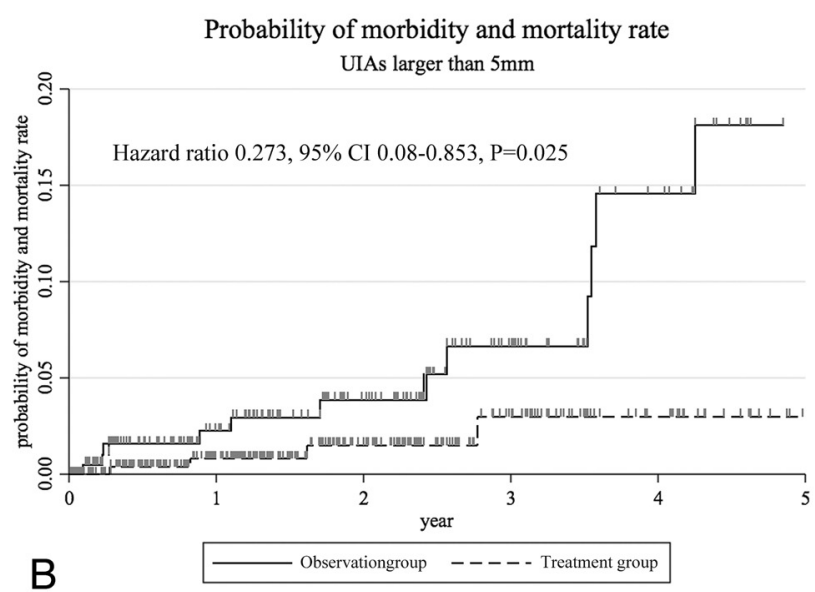

FIG 3. The probability of morbidity and mortality is shown Overall clinical outcomes for the treatment and observation groups are identical for all UIA sizes $(A)$. However, the probability of morbidity and mortality in the treatment group in UIAs larger than $5 \mathrm{~mm}$ is significantly lower than that in the observation group $(P=.025)(B)$. There are no significant differences between the treatment and observation groups in UIAs less than $5 \mathrm{~mm}(C)$. Statistical differences were determined by the Cox proportional hazard model by treatment. morphologic results, and overall clinical outcome and quality of life. However, the study was terminated because of ethical reasons and low patient enrollment. Thus, randomization of therapeutic options for UIAs is nearly impossible.

The size of the UIA was the key factor when preventive treatment was chosen over conservative management. The size of the aneurysm was also related to poor outcome resulting from treatment. Although patients with small $(<5 \mathrm{~mm})$ UIAs had no morbidity and mortality at 30 days after treatment, patients with nonsmall UIAs had a $1.5 \%$ morbidity and mortality rate (Table 5). The rate of neurologic deterioration in our study was $3.4 \%$ (Table 4), consisting of 11 patients with medium or large UIAs. Solomon et $\mathrm{al}^{9}$ reported extremely low morbidity and mortality rates for patients with aneurysms smaller than $10 \mathrm{~mm}$ in diameter. In contrast, surgical outcome of large aneurysms was relatively poor. In the ISUIA, ${ }^{1,2}$ investigators revealed a 2.6 relative risk for poor surgical outcome in aneurysms larger than $12 \mathrm{~mm}$ in diameter.

Overall, our treatment ratio was $33.2 \%$ (Table 1). Therapeutic intervention was equal or superior to conservative management in UIAs of all sizes. Although this study is not randomized, it is the first to compare the risks and morbidity and mortality associated with conservative management of UIAs with those associated with preventive treatment. There was no statistical difference in clinical outcome between the observation group and the treatment group for patients with small $(<5 \mathrm{~mm})$ UIAs, of which we treated only $17.6 \%$. It is uncertain whether UIAs smaller than 5 mm should be treated based on this analysis. Seven small UIAs ruptured during observation, of which 5 were part of multiple aneurysms and 2 were single. On the other hand, our findings showed no clinical adverse events for small aneurysms treated preventively in this study, though the small sample size of the treatment group may have an effect on the data analysis. If patients with small UIAs prefer preventive treatment vs conservative management on the basis of this information, then treatment of these aneurysms may be considered safe and justified. For aneurysms larger than $5 \mathrm{~mm}$, preventive treatment of UIAs was justified if morbidity was defined as an mRS of 3 (moderate disability).

Our study had several limitations. The first limitation was selection bias. Our EVS and MC ratio was almost 90:10 because of patient demand for less invasive treatment. This high trend in EVS was based on the patient referral pattern, and it may interfere as a bias in treatment technique. More than $50 \%$ of the patients in our study were referred from outside of our usual area, and most of these patients chose EVS as the preferred treatment option. If the aneurysmal shape was not suitable for embolization, we recommended MC. However, these patients tended to choose conservative treatment instead of MC. The patients' reasons for refusing our recommendations for treatment were multifactorial, with the most common combination being advanced age, treatment technique preference (EVS over MC), and disagreement among family members.

The second limitation was that our study was not randomized 
and double-blinded. The decision to treat was made mainly by the patient after consultation with the physician and discussion of the results of the study. From an ethical perspective, it was not justifiable to treat very small UIAs or to leave large UIAs untreated for statistical purposes.

The third limitation was the number of patients who were lost to follow-up. Many had relatively small aneurysms (2-4 $\mathrm{mm}$ ) and stopped follow-up after the initial or second visit.

We did not conduct telephone interviews to ask patients the reasons for discontinuing follow-up. However, nearly $90 \%$ of conservatively treated patients continued annual or biannual follow-up; $100 \%$ of treated patients, except those who died or were severely disabled, were followed up. Thus, our single-center study represents the best effort at evaluating the clinical management of UIAs.

The risk for rupture during observation was higher than that reported in the ISUIA, which may be related to our population characteristics or a low dropout rate during follow-up. These data may not be directly applicable to patient populations with different characteristics.

\section{CONCLUSIONS}

Within the limitations of a nonrandomized study, our data suggest that treatment of UIAs was justified in aneurysms larger than $5 \mathrm{~mm}$ and EVS can be safely applied to nearly $90 \%$ of UIAs.

\section{ACKNOWLEDGMENTS}

The authors thank Prof. Mitsuyoshi Urashima at the Division of Clinical Research and Development, the Jikei University School of Medicine, Tokyo, Japan, for help with the statistical evaluation of the data.
Disclosures: Industry Affiliations: Yuichi Murayama and Toshihiro Ishibashi are consultants for Stryker. Yuichi Murayama is a patent holder of Stryker. ICMJE DISCLOSURES: Toshihiro Ishibashi-UNRELATED: Consultancy: Stryker. Hideki Arakawa-UNRELATED: Consultancy: Stryker Japan.

\section{REFERENCES}

1. International Study of Unruptured Intracranial Aneurysms Investigators. Unruptured intracranial aneurysms - risk of rupture and risks of surgical intervention. $N$ Engl J Med 1998;339:1725-33

2. Wiebers DO, Whisnant JP, Huston J 3rd, et al. Unruptured intracranial aneurysms: natural history, clinical outcome, and risks of surgical and endovascular treatment. Lancet 2003;362:103-10

3. Ishibashi T, Murayama Y, Urashima M, et al. Unruptured intracranial aneurysms: incidence of rupture and risk factors. Stroke 2009;40:313-16

4. UCAS Japan Investigators, Morita A, Kirino $\mathrm{T}$, et al. The natural course of unruptured cerebral aneurysms in a Japanese cohort. N Engl J Med 2012:366:2747-82

5. Japanese Society for Detection of Asymptomatic Brain Diseases. Guidelines for UIA. http://jbds.jp/guideline.html. Accessed on May 4, 2012

6. Murayama Y, Saguchi T, Ishibashi T, et al. Endovascular operating suite: future directions for treating neurovascular disease. J Neurosurg 2006;104:925-30

7. Takao H, Murayama Y, Ishibashi T, et al. Comparing accuracy of cerebral aneurysm size measurements from three routine investigations: computed tomography, magnetic resonance imaging, and digital subtraction angiography. Neurol Med Chir (Tokyo) 2010;50:893-99

8. Raymond D, Darsaut TE, Molyneux AJ, TEAM Collaborative Group. A trial on unruptured intracranial aneurysms (the TEAM trial): results, lessons from a failure and the necessity for clinical care trials. Trials 2011;12:64

9. Solomon RA, Fink ME, Pile-Spellman J. Surgical management of unruptured intracranial aneurysms. J Neurosurg 1994:80:440-46 